\title{
Une boîte à outils pour les médecins
}

\section{Stefan Ritler}

Vice-directeur Office fédéral des assurances sociales OFAS, chef du domaine AI

Un traitement médical adéquat est un facteur essentiel à la réussite de la réadaptation sociale et professionnelle des personnes atteintes dans leur santé. Une coordination de ce traitement avec les mesures d'ordre professionnel de l'assuranceinvalidité (AI) est toutefois indispensable. Cela suppose que le médecin traitant et le personnel de l'office AI et du service médical régional de l'AI travaillent en étroite collaboration.

Une action rapide revêt une importance toute particulière dans le cas des personnes malades qui ont encore un emploi ou une place de formation. Le médecin traitant est alors en première ligne. Il est le mieux placé pour juger de la capacité de travail de son patient et pour identifier les déficits qui subsisteront éventuellement lors de la réadaptation. S'il éprouve des difficultés à déterminer l'incapacité de travail d'un patient, il peut, d'entente avec ce dernier et indépendamment de l'AI, prendre contact avec l'employeur ou l'institution de formation. Le médecin traitant a ainsi la possibilité, avant de rédiger le certificat médical, de mieux connaître les exigences auxquelles son patient est exposé sur son lieu de travail.

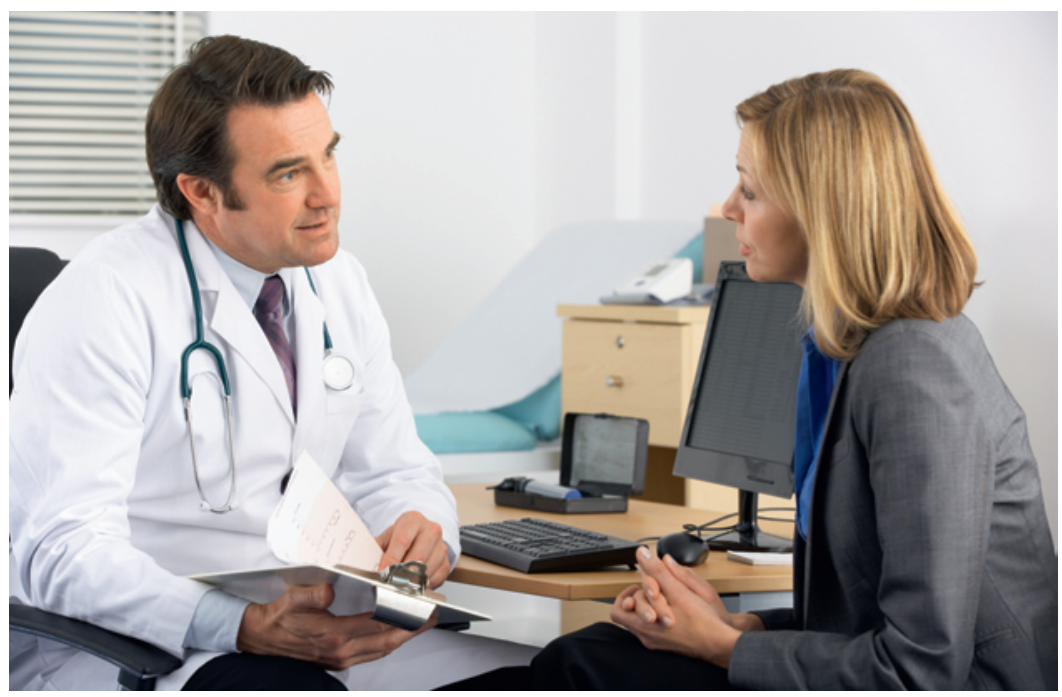

Indispensable à une réadaptation sociale et professionnelle des personnes atteintes dans leur santé: l'échange intensif entre médecins traitants et le personnel de l'office Al (image prétexte).
Pour améliorer l'évaluation de la capacité de travail, la société des médecins du canton de Saint-Gall et les associations d'employeurs du canton ont mis au point un certificat médical détaillé et un formulaire de description du poste de travail à remplir par l'employeur

Le Centre de formation AI offre désormais aux médecins hospitaliers et aux autres médecins un cours gratuit qui donne une vue d'ensemble du système de sécurité sociale en Suisse et du fonctionnement de l'AI.

(Wiedersheim, 2014). Ce certificat médical a été ajouté aux modèles proposés par Swiss Insurance Medicine sur son site Internet (lien: www.swiss-insurance-medi cine.ch). L'association Compasso, placée sous le patronage de l'Union patronale suisse, travaille actuellement à perfectionner ce document avec l'ensemble des acteurs concernés. Le nouveau certificat médical sera rendu public en 2018. La plateforme d'information www.compasso.ch contient également des informations qui intéressent les médecins traitants.

\section{Le médecin traitant comme partenaire central de l'AI}

Le médecin traitant et l'office AI ont un intérêt commun à ce que la personne atteinte dans sa santé puisse, malgré son absence pour cause de maladie, conserver sa place de travail ou retrouver une activité adaptée. Dès lors qu'une personne est restreinte dans sa capacité de travail, il est souhaitable que le médecin traitant, d'entente avec elle, prenne immédiatement contact avec l'office AI compétent. Celui-ci convoque alors la per- 
sonne concernée pour un premier entretien, si possible avec le médecin traitant et l'employeur, et clarifie sa situation médicale, sociale et professionnelle avec l'ensemble des participants. Ce premier entretien débouche, selon les résultats, soit sur le dépôt d'une demande de prestations AI, soit sur l'orientation vers une consultation sociale ou un autre service mieux à même d'apporter un soutien à l'assuré. Lorsqu'une demande de prestations AI est déposée, les offices AI sollicitent des informations auprès de l'assureur-maladie, de l'assureur d'indemnités journalières, des hôpitaux et des employeurs. Le recours à la documentation existante et le contact direct avec le médecin traitant peut éviter aux offices AI d'avoir à solliciter un rapport du médecin traitant ou lui permettre de ne demander qu'un rapport couvrant les questions encore en suspens. Un nouveau formulaire, présenté sous forme de modules, sera disponible début 2018 et permettra aux offices AI de poser au médecin uniquement les questions nécessaires.

Le médecin traitant peut facturer ses prestations à l'AI, y compris la rédaction d'un rapport médical ou la participation aux réunions. Il trouvera sur la plateforme ai-pro-medico.ch, administrée conjointement par l'AI et la FMH, des informations sur le fonctionnement des offices AI, sur le déroulement d'un processus de réadaptation, sur les modalités de facturation à l'office AI et sur bien d'autres points.

\section{Echange mutuel d'informations et offres de formation de l'AI}

La réussite de la réadaptation d'une personne atteinte dans sa santé suppose une coordination du traitement médical et des efforts de réadaptation professionnelle. Concrètement, cela signifie que la collaboration entre l'office AI et le médecin traitant doit toujours chercher à fournir à cette personne une prestation simple, pertinente et de qualité. Afin qu'il puisse adapter ses prestations au processus de réadaptation, le médecin traitant a besoin d'informations sur la situation actuelle de son patient dans la procédure AI. Cela n'est pas possible actuellement. Pour y remédier, la réforme «Développement continu de l'AI» prévoit que les collaborateurs de l'AI puissent fournir des informations aux médecins traitants. L'échange mutuel d'informations s'en trouvera facilité, conformément à une demande formulée de longue date par les deux parties. Voir à ce sujet l'article «Développement continu de l'AI: aperçu des principales mesures» dans le présent numéro.

Le Centre de formation AI offre désormais aux médecins hospitaliers et aux autres médecins un cours gratuit qui donne une vue d'ensemble du système de sécurité sociale en Suisse et du fonctionnement de l'AI. Une autre question abordée est la valeur des rapports médicaux et la façon dont ces rapports et les prescriptions de conseil des médecins traitants doivent être facturés à l'AI. Enfin, le cours entend donner aux médecins traitants une plus grande sécurité dans l'évaluation de la capacité de travail de leurs patients. D'autres cours sont répertoriés dans le catalogue annuel du centre de formation à l'adresse www.cfai.ch. Une inscription est nécessaire et peut être effectuée en ligne depuis cette adresse Internet.

Les mesures prévues dans le cadre du développement continu de l'AI et les informations disponibles sur le site www.ai-pro-medico.ch visent à répondre au reproche d'opacité parfois formulé contre l'AI, mais aussi à simplifier et à renforcer la collaboration entre l'AI et les médecins traitants.

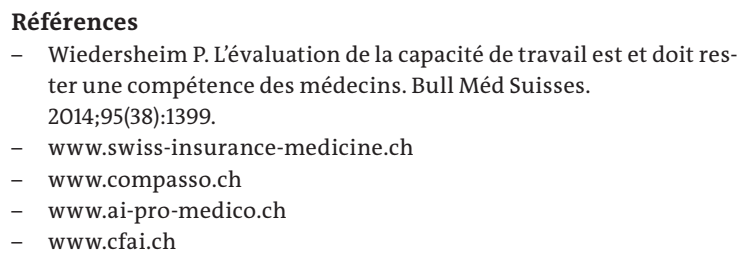

Crédit photo

(c) Monkeybusinessimages | Dreamstime.com 